\title{
EUCLIDEAN WINDOWS
}

\section{STEFANIA CAVALLAR AND FRANZ LEMMERMEYER}

\begin{abstract}
In this paper we study number fields which are Euclidean with respect to functions that are different from the absolute value of the norm, namely weighted norms that depend on a real parameter $c$. We introduce the Euclidean minimum of weighted norms as the set of values of $c$ for which the function is Euclidean, and we show that the Euclidean minimum may be irrational and not isolated. We also present computational results on Euclidean minima of cubic number fields, and present a list of norm-Euclidean complex cubic fields that we conjecture to be complete.
\end{abstract}

\section{Introduction}

Let $R$ be an integral domain. A function $f: R \longrightarrow \mathbb{R}_{\geqslant 0}$ is called a Euclidean function on $R$ if it satisfies the following conditions with $\kappa=1$ :

(i) $f(R) \cap[0, c]$ is finite for every $c \geqslant 0$;

(ii) $f(r)=0$ if and only if $r=0$;

(iii) for all $a, b \in R$ with $b \neq 0$ there exists a $q \in R$ such that $f(a-b q)<\kappa \cdot f(b)$.

If $f: R \longrightarrow \mathbb{R}_{k 00}$ is a function satisfying conditions (i) and (ii), then the infimum of all $\kappa \in \mathbb{R}$ such that condition (iii) holds is called the Euclidean minimum of $R$ with respect to $f$, and will be denoted by $M(R, f)$; thus for all $a, b \in R \backslash\{0\}$ and every $\varepsilon>0$ there is a $q \in R$ (possibly depending on $\varepsilon$ ) such that $f(a-b q)<M(R, f) \cdot f(b)+\varepsilon$.

If $f$ is a multiplicative function, then we can replace condition (iii) by the equivalent condition that for every $\xi \in K$ ( $K$ being the quotient field of $R$ ) there is a $q \in R$ such that $f(\xi-q)<\kappa$. The infimum of all $\kappa \in \mathbb{R}$ such that this condition holds for a fixed $\xi$ is denoted by $M(\xi, f)$; clearly $M(R, f)$ is the supremum of the $M(\xi, f)$.

If $R=\mathcal{O}_{K}$ is the ring of integers in a number field $K$, then the absolute value of the norm satisfies conditions (i) and (ii), and a folklore conjecture states that $M(K):=M(R,|N|)$ coincides with the inhomogeneous minimum of the norm form of $\mathcal{O}_{K}$. (This conjecture is known to hold for number fields with unit rank at most 1.) Let $C_{1}$ be a set of representatives modulo $\mathcal{O}_{K}$ of all $\xi=a / b \in K$ with $M(\xi)=M(K)$ (here $M(\xi):=M(\xi,|N|)$ ); then we say that $M(K)$ is isolated if there is a $\kappa_{2}<\kappa$ such that $M(\xi) \leqslant \kappa_{2}$ for all $\xi \in K$ that are not represented by some point in $C_{1}$. In that case, we define the second minimum $M_{2}(K)$ (as well as higher minima $\left.M_{k}(R)\right)$ as in [13] or [6] by considering only the minima of points that are not represented by points in $C_{1}$, and so on.

Replacing $K$ in these definitions by $\bar{K}=\mathbb{R}^{n}$ (this is the topological closure of the image of $K$ under the standard embedding $K \longrightarrow \mathbb{R}^{n}$; for totally real fields we have $\bar{K}=K \otimes_{\mathbb{Q}} \mathbb{R}$ ), the Euclidean minimum becomes the inhomogeneous minimum of the norm form of $K$; 
we clearly have $M_{j}(\bar{K}) \geqslant M_{j}(K)$ whenever these minima are defined, and it is conjectured that $M_{1}(\bar{K})=M_{1}(K)$ is rational.

The aim of this paper is to explain how the Euclidean minimum of $\mathcal{O}_{K}$ with respect to 'weighted norms' can be computed in some cases; we shall show that the Euclidean minimum for certain weighted norms in $\mathbb{Q}(\sqrt{69})$ is irrational and not isolated, thereby showing that these conjectured properties for minima with respect to the usual norm do not carry over to weighted norms.

\section{Weighted norms}

Let $K$ be a number field, $\mathcal{O}_{K}$ its ring of integers, and $\mathfrak{p}$ a prime ideal in $\mathcal{O}_{K}$. Then, for any real number $c>0$,

$$
\phi: \mathfrak{q} \longmapsto \begin{cases}N \mathfrak{q}, & \text { if } \mathfrak{q} \neq \mathfrak{p}, \\ c, & \text { if } \mathfrak{q}=\mathfrak{p},\end{cases}
$$

defines a map from the set of prime ideals $\mathfrak{q}$ of $\mathcal{O}_{K}$ into the positive real numbers, which can be uniquely extended to a multiplicative map $\phi: I_{K} \longrightarrow \mathbb{R}_{>0}$ on the group $I_{K}$ of fractional ideals. Putting $f(\alpha)=\phi\left(\alpha \mathcal{O}_{K}\right)$ for any $\alpha \in K^{\times}$and $f(0)=0$, we get a function $f=f_{\mathfrak{p}, c}: K \longrightarrow \mathbb{R}_{\geqslant 0}$ which $\mathrm{H}$. W. Lenstra [14] called a weighted norm.

Our aim is to study examples of number fields that are Euclidean with respect to some weighted norm. Lenstra [14] showed that $\mathbb{Q}\left(\zeta_{3}\right)$ and $\mathbb{Q}\left(\zeta_{4}\right)$ are such fields, but the first examples that are not norm-Euclidean were given by D. Clark $[7,8]$.

A formal condition for $f_{\mathfrak{p}, c}$ to be a Euclidean function is the finiteness of the sets $\left\{f_{\mathfrak{p}, c}(\alpha)<\lambda: \alpha \in \mathcal{O}_{K}\right\}$ for all $\lambda \in \mathbb{R}$. This property is easily seen to be equivalent to $c>1$.

For weighted norms $f=f_{\mathfrak{p}, c}$ on $K$, we define (following [13]) the Euclidean window of $\mathfrak{p}, w(\mathfrak{p})$, by

$$
w(\mathfrak{p})=\left\{c \in \mathbb{R}: f_{\mathfrak{p}, c} \text { is a Euclidean function on } \mathcal{O}_{K}\right\} .
$$

Proposition 1.1. The Euclidean window is a (possibly empty) interval contained in $(1, \infty)$.

Proof. Assume that $w(\mathfrak{p})$ is not empty, and let $r, t \in w(\mathfrak{p})$ with $r<t$. Then it is sufficient to show that $f_{\mathfrak{p}, s}$ is a Euclidean function on $\mathcal{O}_{K}$ for every $r \leqslant s \leqslant t$. Now $\mathcal{O}_{K}$ is Euclidean with respect, for example, to $f_{\mathfrak{p}, r}$, so $\mathcal{O}_{K}$ is a principal ideal domain; hence every $\xi \in K$ has the form $\xi=\alpha / \beta$ with $(\alpha, \beta)=1$. Moreover, there exist $\gamma_{r}, \gamma_{t} \in \mathcal{O}_{K}$ such that

$$
f_{\mathfrak{p}, r}\left(\alpha-\beta \gamma_{r}\right)<f_{\mathfrak{p}, r}(\beta), \quad f_{\mathfrak{p}, t}\left(\alpha-\beta \gamma_{t}\right)<f_{\mathfrak{p}, t}(\beta) .
$$

If $\mathfrak{p} \nmid \beta$, then

$$
f_{\mathfrak{p}, s}\left(\alpha-\beta \gamma_{t}\right) \leqslant f_{\mathfrak{p}, t}\left(\alpha-\beta \gamma_{t}\right)<f_{\mathfrak{p}, t}(\beta)=f_{\mathfrak{p}, s}(\beta) ;
$$

if $\mathfrak{p} \mid \beta$, on the other hand, then $\mathfrak{p} \nmid \alpha$, and hence $\mathfrak{p} \nmid\left(\alpha-\beta \gamma_{r}\right)$, and

$$
f_{\mathfrak{p}, s}\left(\alpha-\beta \gamma_{r}\right)=f_{\mathfrak{p}, r}\left(\alpha-\beta \gamma_{r}\right)<f_{\mathfrak{p}, r}(\beta) \leqslant f_{\mathfrak{p}, s}(\beta) .
$$

Thus $f_{\mathfrak{p}, s}$ is indeed a Euclidean function on $\mathcal{O}_{K}$.

In this paper, we investigate Euclidean windows for various algorithms in some quadratic and cubic number fields; we shall give examples of empty, finite and infinite Euclidean windows, and we show that the first minima with respect to weighted norms need not be rational. First, however, we shall show that the Euclidean minima for weighted norms need not be continuous functions of $c$ for rings of integers with finite unit groups. 


\section{Euclidean windows}

\section{Weighted norms in $\mathbb{Z}$}

The Euclidean window for primes in $\mathbb{Z}$ can easily be determined, as follows.

Proposition 2.1. The Euclidean minimum $M\left(f_{p, c}\right)$ of a weighted norm in $\mathbb{Z}$ is given by

$$
M\left(f_{p, c}\right)= \begin{cases}\infty & \text { if } c<p, \\ \frac{1}{2} & \text { if } c=p, \\ 1 & \text { if } c>p .\end{cases}
$$

Moreover, $w(p)=[p, \infty)$.

Proof. We first show that $M\left(f_{p, c}\right)=\infty$ if $c<p$ (this implies that $w(p) \subseteq[p, \infty)$ ). To this end, put $b=p^{n}$ and

$$
a= \begin{cases}\frac{1}{2}\left(p^{n}-1\right) & \text { if } p \neq 2 \\ 2^{n-1}-1 & \text { if } p=2\end{cases}
$$

Then $p \nmid(a-b q)$; hence $f_{p, c}(a-b q)=|a-b q|$ for all $q \in \mathbb{Z}$. If the minimum $\kappa=M\left(f_{p, c}\right)$ were finite, there would exist a $q \in \mathbb{Z}$ such that $f_{p, c}(a-b q)<\kappa f_{p, c}(b)=\kappa c^{n}$. But clearly $|a| \leqslant|a-b q|=f_{p, c}(a-b q)$, and hence we get $|a| c^{-n}<\kappa$ for all $n \in \mathbb{N}$; however, since $c<p$, the expression on the left-hand side tends to $\infty$ with $n$.

Since it is well known that $M\left(f_{p, p}\right)=12$, we next show that $M\left(f_{p, c}\right)=1$ if $c>p$. To this end, choose $\alpha, \beta \in \mathbb{N}$, not divisible by $p$, such that $p<\alpha / \beta<c$. If we put $a=p^{n} \beta^{n}$ and $b=\alpha^{n}+p^{n} \beta^{n}$, then we get

$$
\begin{aligned}
f_{p, c}\left(\frac{a}{b}\right) & =\frac{c^{n} \beta^{n}}{\alpha^{n}+p^{n} \beta^{n}}=\frac{c^{n}}{(\alpha / \beta)^{n}+p^{n}}>\frac{c^{n}}{c^{n}+p^{n}}, \\
f_{p, c}\left(\frac{a}{b}-1\right) & =\frac{\alpha^{n}}{\alpha^{n}+p^{n} \beta^{n}},
\end{aligned}
$$

and both expressions tend to 1 as $n$ goes to $\infty$. Note also that $f_{p, c}(a / b-q) \geqslant|a / b-q|>1$ for all $q \in \mathbb{N} \backslash\{0,1\}$, since the denominator of $(a / b-q)$ is prime to $p$, and since $c>p$.

Thus $M\left(f_{p, c}\right) \geqslant 1$ if $c>p$; but we can easily show that $M\left(f_{p, c}\right) \leqslant 1$ by proving that $f_{p, c}$ is a Euclidean function for all $p \geqslant c$. In fact, suppose that $a, b \in \mathbb{Z} \backslash\{0\}$ are given, and that they are relatively prime. If $p \mid b$, then $p \nmid(a-b q)$ for all $q \in \mathbb{Z}$; hence $f_{p, c}(a-b q)=|a-b q|$, and we can certainly find $q \in \mathbb{Z}$ such that $|a-b q|<|b|$. But $|b| \leqslant f_{p, c}(b)$ since $c \geqslant p$.

Now consider the case $p \nmid b$; then we choose $q \in \mathbb{Z}$ such that $|a-b q|,|a-b(q+1)| \leqslant b$. But $r=a-b q$ and $r^{\prime}=a-b(q+1)$ cannot both be divisible by $p$; if $p \nmid r$, then $f_{p, c}(r)=|r|<|b|=f_{p, c}(b)$, and if $p \nmid r^{\prime}$, then $f_{p, c}\left(r^{\prime}\right)<f_{p, c}(b)$.

\section{Weighted norms in $\mathbb{Q}(\sqrt{14})$}

Since it is well known that an imaginary quadratic number field is Euclidean if and only if it is norm-Euclidean, only the case of real quadratic fields is interesting. We shall deal with only two examples here: one is $\mathbb{Q}(\sqrt{14})$, which has been studied often in this respect (see the work of Bedocchi [2], Nagata $[15,16]$ and Cardon [3]), and the other is $\mathbb{Q}(\sqrt{69})$, which was shown to be Euclidean with respect to a weighted norm by Clark [7] (see also work by Niklasch [17] and Hainke [11]); $\mathbb{Q}(\sqrt{69})$ is discussed in Sections 4 and 5. 
Consider the quadratic number field $K=\mathbb{Q}(\sqrt{14})$. It is well known that $M_{1}(K)=$ $5 / 4$ and $M_{2}(K)=31 / 32$ (see [13]); moreover, $M_{1}$ is attained exactly at the points $\xi \equiv(1+\sqrt{14}) / 2 \bmod \mathcal{O}_{K}$. Now we claim that the following proposition is true.

Proposition 3.1. For $K=\mathbb{Q}(\sqrt{14})$ and $\mathfrak{p}=(2, \sqrt{14})$ we have $w(\mathfrak{p}) \subseteq(\sqrt{5}, \sqrt{7})$.

Proof. Put $\alpha=1+\sqrt{14}$, and $\beta=2$. Then $|N(\alpha-\beta \gamma)|$ is an odd integer greater than or equal to 5 for all $\gamma \in \mathcal{O}_{K}$. Thus $f_{\mathfrak{p}, c}(\alpha-\beta \gamma)=|N(\alpha-\beta \gamma)| \geqslant 5$, and if $f_{\mathfrak{p}, c}$ is a Euclidean function, we must have $5<f_{\mathfrak{p}, c}(\beta)=c^{2}$. This shows that $c>\sqrt{5}$.

In order to show that $c<\sqrt{7}$ we look at the ideal $\mathfrak{q}=(7, \sqrt{14})=(7+2 \sqrt{14})$ of norm 7. If $f_{\mathfrak{p}, c}$ is Euclidean, then every residue class modulo $\mathfrak{q}$ must contain an element $\alpha$ such that $f_{\mathfrak{p}, c}(\alpha)<f_{\mathfrak{p}, c}(\mathfrak{q})=7$.

Since the unit group generates the subgroup $\{-1,+1\}$ of $\left(\mathcal{O}_{K} / \mathfrak{q}\right)^{\times}\left(\right.$and $\left.f_{\mathfrak{p}, c}( \pm 1)=1\right)$, and since $\pm 3+\sqrt{14} \equiv \pm 3 \bmod \mathfrak{q}\left(\right.$ where $\left.f_{\mathfrak{p}, c}( \pm 3+\sqrt{14})=|N( \pm 3+\sqrt{14})|=5\right)$, we must find elements in the residue classes $\pm 2 \bmod \mathfrak{q}$. The only possible candidates are powers of $4+\sqrt{14}$, because the only ideals of odd norm less than 7 are $(0),(1)$, and $(3 \pm \sqrt{14})$, none of which yields elements equivalent to $\pm 2 \bmod \mathfrak{q}$. Moreover, $\pm 4+\sqrt{14} \equiv \pm 3 \bmod \mathfrak{q}$, and we see that if there exist elements $\alpha \equiv 2 \bmod \mathfrak{q}$ with $f_{\mathfrak{p}, c}(\alpha)<7$, then $\alpha=2$ is one of them. But $f_{\mathfrak{p}, c}(2)=c^{2}$, and we find that $c<\sqrt{7}$.

We remark that it is not known whether $w(\mathfrak{p})$ is empty or not.

If we look at prime ideals other than $(2, \sqrt{14})$, the situation is quite different.

Proposition 3.2. Let $K=\mathbb{Q}(\sqrt{14})$, and let $\mathfrak{p}$ be a prime ideal in $\mathcal{O}_{K}$ of norm $N \mathfrak{p} \equiv$ $\pm 1 \bmod 8$. Then $w(\mathfrak{p})=\varnothing$.

Proof. Assume that $f_{\mathfrak{p}, c}$ is a Euclidean function. Then there exists an

$$
\alpha=x+y \sqrt{14} \equiv 1+\sqrt{14} \bmod 2
$$

such that $f_{\mathfrak{p}, c}(\alpha)<f_{\mathfrak{p}, c}(2)=4$. Since $\alpha$ cannot be a unit, this is possible only if $\alpha$ is divisible by $\mathfrak{p}$. If $\alpha$ is divisible by some other prime ideal $\mathfrak{q}$, then $f_{\mathfrak{p}, c}(\mathfrak{q})=N \mathfrak{q} \geqslant 5$, and we conclude that $f_{\mathfrak{p}, c}(\mathfrak{p})<1$, a contradiction. Thus $(\alpha)=\mathfrak{p}^{m}$ for some $m \geqslant 1$. But $\mathfrak{p}=(a+b \sqrt{14})$ since $K$ has class number 1 , and $b$ must be even since $\pm p=a^{2}-14 b^{2} \equiv \pm 1 \bmod 8$ : thus $a+b \sqrt{14} \not \equiv 1+\sqrt{14} \bmod 2$, and again we have a contradiction.

\section{The Euclidean algorithm in $\mathbb{Q}(\sqrt{69})$}

Next we study the field $\mathbb{Q}(\sqrt{69})$; we shall prove the following result, which corrects a claim announced without proof in [13], namely that $M_{2}(K)<M_{2}(\bar{K})$, and that $M_{2}(\bar{K})$ is isolated.

Theorem 4.1. In $K=\mathbb{Q}(\sqrt{69})$, we have

$$
\begin{array}{ll}
M_{1}=\frac{25}{23}, & C_{1}=\left\{ \pm \frac{4}{23} \sqrt{69}\right\}, \\
M_{2}=\frac{15}{46}(11-\sqrt{69}), & C_{2}=\left\{\left( \pm P_{r}, \pm P_{r}^{\prime}\right)\right\}, r \geqslant 0
\end{array}
$$

where

$$
P_{r}=\frac{1}{2} \varepsilon^{-r}+\left(\frac{4}{23}+\frac{1}{2 \sqrt{69}} \varepsilon^{-r}\right) \sqrt{69}, \quad P_{r}^{\prime}=\frac{1}{2} \varepsilon^{-r}-\left(\frac{4}{23}+\frac{1}{2 \sqrt{69}} \varepsilon^{-r}\right) \sqrt{69}
$$


Here $M_{j}$ denotes the $j$ th inhomogeneous minimum of the norm form of $\mathcal{O}_{K}, C_{j}$ is a set of representatives modulo $\mathcal{O}_{K}$ of the points where $M_{j}$ is attained, and $\varepsilon=(25+3 \sqrt{69}) / 2$ is the fundamental unit of $K$. The second minimum, $M_{2}(K)=M_{2}(\bar{K})$, is not isolated.

The proof of Theorem 4.1 is based on methods developed by Barnes and SwinnertonDyer [1]. In the discussion that follows, we shall regard $K$ as a subset of $\mathbb{R}^{2}$ via the embedding $(x+y \sqrt{69}) \longrightarrow(x, y)$. Conversely, any point $P=(x, y) \in \mathbb{R}^{2}=\bar{K}$ corresponds to a pair $\xi_{P}=x+y \sqrt{69}, \xi_{P}^{\prime}=x-y \sqrt{69}$. These elements are not necessarily in $K$; nevertheless, we call $\xi_{P}^{\prime}=x-y \sqrt{69}$ the conjugate of $\xi_{P}$. Note, for example, that $\xi_{P}=\sqrt{69}$ alone does not determine $P$, since both $P=(0,1)$ and $P=(\sqrt{69}, 0)$ correspond to such a $\xi_{P}$. The ' $\bar{K}$-valuations' $\left.|\cdot|\right|_{1}$ and $\left.|\cdot|\right|_{2}$ are defined by $|(x, y)|_{1}=|x+y \sqrt{69}|$ and $|(x, y)|_{2}=|x-y \sqrt{69}|$, with a positive square root of 69 .

Using the technique described in [6], it is easy to cover the whole fundamental domain of the lattice $\mathcal{O}_{K}$ with a bound of $k=0.875$, except for $\pm S_{0} \cup \pm S_{1} \cup \pm S_{2} \cup \pm T$, where

$$
\begin{aligned}
& S_{0}=\left[\begin{array}{ll}
-0.00085, & 0.00085
\end{array}\right] \times[0.1739,0.1742] ; \\
& S_{1}=\left[\begin{array}{lll}
0.01917, & 0.02005] & \times
\end{array}\right][0.1763,0.1765] ; \\
& S_{2}=[-0.02005,-0.01917] \times[0.1763,0.1765] ; \\
& T=[0.4999,0.5001] \times[0.2341,0.2342] .
\end{aligned}
$$

We find, transforming these exceptional sets by multiplication with the units $\varepsilon$ and $\bar{\varepsilon}=(25-3 \sqrt{69}) / 2$, for example, that

$$
\varepsilon S_{0} \subset 18+2 \sqrt{69}+[-0.012,0.041] \times[0.172,0.179]
$$

that is, $\varepsilon S_{0}-(18+2 \sqrt{69})$ is contained in covered regions or $S_{0} \cup S_{1}$, which we shall denote by $\varepsilon S_{0}-(18+2 \sqrt{69}) \widetilde{\subset} S_{0} \cup S_{1}$. Similar calculations show that

$$
\begin{array}{llllll}
\varepsilon S_{0}-(18+2 \sqrt{69}) & \widetilde{\complement} & S_{0} \cup S_{1} ; & \bar{\varepsilon} S_{0}+(18-2 \sqrt{69}) & \widetilde{\complement} & S_{0} \cup S_{2} ; \\
\varepsilon S_{1}-(18+2 \sqrt{69}) & \widetilde{\simeq} & T ; & \bar{\varepsilon} S_{1}+(18-2 \sqrt{69}) & \widetilde{\simeq} & S_{0} \cup S_{2} ; \\
\varepsilon S_{2}-(18+2 \sqrt{69}) & \widetilde{\complement} & S_{0} \cup S_{1} ; & \bar{\varepsilon} S_{2}+(19-2 \sqrt{69}) & \widetilde{\complement} & T ; \\
\varepsilon T-(61+7 \sqrt{69}) / 2 & \widetilde{\complement} & S_{2} ; & \bar{\varepsilon} T+(18-2 \sqrt{69}) & \widetilde{\complement} & S_{1} .
\end{array}
$$

Remark. The inclusions on the right-hand side can be computed from those on the left: for example, all exceptional points in $S_{2}$ must come from $T$, so the exceptional points in $\varepsilon^{-1} S_{2}$ must be congruent modulo $\mathcal{O}_{K}$ to points in $T$, and since $(61+7 \sqrt{69}) / 2 \varepsilon=19-2 \sqrt{69}$, we conclude that $\bar{\varepsilon} S_{2}+(19-2 \sqrt{69}) \widetilde{\subset} T$.

We shall need the following result (this is [6, Proposition 2]).

Proposition 4.2. Let $K$ be a number field, and $\varepsilon$ a non-torsion unit of $E_{K}$. Suppose that $S \subset \widetilde{F}$ has the following property.

There exists a unique $\theta \in \mathcal{O}_{K}$ such that, for all $\xi \in S$, the element $\varepsilon \xi-\theta$ lies in a $k$-covered region of $\widetilde{F}$, or again in $S$.

Then every $k$-exceptional point $\xi_{0} \in S$ satisfies $\left|\xi_{0}-\theta /(\varepsilon-1)\right|_{j}=0$ for every $\bar{K}$-valuation $|\cdot|_{j}$ such that $|\varepsilon|_{j}>1$.

We also need a method to compute Euclidean minima of given points. Recall that the orbit of $\xi \in \bar{K}$ is the $\operatorname{set} \operatorname{Orb}(\xi)=\left\{\varepsilon \xi: \varepsilon \in E_{K}\right\}$, where $E_{K}$ is the unit group of $\mathcal{O}_{K}$. Note that all the elements in an orbit have the same minimum. 
Proposition 4.3. Let $m \in \mathbb{N}$ be square-free, let $K=\mathbb{Q}(\sqrt{m})$ be a real quadratic number field, let $\varepsilon>1$ be a unit in $\mathcal{O}_{K}$, and let $\xi \in \bar{K}$. If $M(K, \xi)<k$ for some real $k$, then there exists an element $\eta=r+s \sqrt{m} \in K$ with the following properties.

(i) $\eta \equiv \xi_{j} \bmod \mathcal{O}_{K}$ for some $\xi_{j} \in \operatorname{Orb}(\xi)$.

(ii) $|N \eta|<k$.

(iii) $|r|<\mu,|s|<\mu / \sqrt{m}$, where $\mu=(\sqrt{k} / 2)(\sqrt{\varepsilon}+1 / \sqrt{\varepsilon})$.

Proof. Assume that $M(K, \xi)<k$; then there is an $\alpha \in \mathcal{O}_{K}$ such that $|N(\xi-\alpha)|<k$. Choose $m \in \mathbb{Z}$ such that $\sqrt{k / \varepsilon} \leqslant\left|(\xi-\alpha) \varepsilon^{m}\right|<\sqrt{k \varepsilon}$, and put $\eta=(\xi-\alpha) \varepsilon^{m}$. Then we prove the three parts of the proposition as follows.

(i) $\eta=(\xi-\alpha) \varepsilon^{m} \equiv \xi \varepsilon^{m} \bmod \mathcal{O}_{K}$, and clearly $\xi^{m} \in \operatorname{Orb}(\xi)$.

(ii) $|N \eta|=|N(\xi-\alpha)|<k$.

(iii) Write $\eta=r+s \sqrt{m}$ and $\eta^{\prime}=r-s \sqrt{m}$. Then $|\eta|<\sqrt{k \varepsilon}$ and $\left|\eta^{\prime}\right|=\left|\eta \eta^{\prime}\right| /|\eta|<$ $k /|\eta| \leqslant \sqrt{k \varepsilon}$. Thus $2|r|=\left|\eta+\eta^{\prime}\right| \leqslant|\eta|+\left|\eta^{\prime}\right|$ and $2|s| \sqrt{m}=\left|\eta-\eta^{\prime}\right| \leqslant|\eta|+\left|\eta^{\prime}\right|$. Using the lemma below, this yields the desired bounds.

This concludes the proof.

Lemma 4.4. If $x, y$ are positive real numbers such that $x<a, y<a$ and $x y<b$, then $x+y<a+b / a$.

Proof. $0<(a-x)(a-y)=a^{2}-a(x+y)+x y<a^{2}-a(x+y)+b$.

Now we are ready to determine a certain class of exceptional points inside $S_{0}$.

Claim 4.1. If $P$ is an exceptional point in $S_{0}$ that stays inside $S_{0}$ under repeated applications of the maps

$$
\begin{aligned}
& \alpha: \xi \longmapsto \varepsilon^{-1} \xi+18-2 \sqrt{69}, \\
& \beta: \xi \longmapsto \varepsilon \xi-(18+2 \sqrt{69}),
\end{aligned}
$$

then $P=(18+2 \sqrt{69}) /(\varepsilon-1)=(0,4 / 23)$. Moreover, $M(P)=25 / 23$.

This follows directly from Proposition 4.2; the Euclidean minimum $M(P)=25 / 23$ is easily computed using Proposition 4.3. Any exceptional point that does not stay inside $S_{0}$ must eventually come through $T$; it is therefore sufficient to consider exceptional points in $T$ from now on.

Let $P_{0} \in T$ be such an exceptional point, and define the series of points $P_{0}, P_{1}, P_{2}, \ldots$ recursively by $P_{j+1}=\alpha\left(P_{j}\right)$. Then $P_{1} \in S_{1}$, and now there are the following two possibilities.

(A) $P_{j} \in S_{0}$ for all $j \geqslant 2$.

(B) There is an $n \geqslant 2$ such that $P_{n} \in S_{1}$.

Before we can go in the other direction, we have to adjust $P_{0}$ somewhat. In fact, $\beta\left(P_{0}\right) \in$ $T$ implies that $\beta\left(P_{0}\right)-\varepsilon \widetilde{\subset} S_{2}$; thus we can define a sequence of points $P_{0}-1, P_{-1}, P_{-2}, \ldots$ by $P_{-1}=\beta\left(P_{0}-1\right)$ and $P_{-j-1}=\beta\left(P_{-j}\right)$ for $j \geqslant 1$. Again, there are two possibilities.

(C) $P_{-j} \in S_{0}$ for all $j \geqslant 2$.

(D) There is an $n \geqslant 2$ such that $P_{-n} \in S_{1}$. 


\section{Euclidean windows}

Claim 4.2. If $P_{0} \in T$ is an exceptional point satisfying conditions (A) and (C), then

$$
P_{0}=\left(\frac{1}{2}, \frac{4}{23}+\frac{1}{2 \sqrt{69}}\right) \approx(0.5,0.234105)
$$

Note that this point is not contained in $K$. Of course, we knew this before: every point in $K$ has a finite orbit, whereas $P_{0}$ does not.

For a proof, we apply Proposition 4.2 to the set $S=\left\{P_{0}, P_{1}, P_{2}, \ldots\right\}$; this shows that any $\xi=P_{j}$ lies on the line $|\xi+(18-2 \sqrt{69}) /(\bar{\varepsilon}-1)|_{2}=0$ (the $\bar{K}$-valuation $|\cdot|_{2}$ is chosen so that $\left.|\bar{\varepsilon}|_{2}>1\right)$; that is, $\xi^{\prime}=-(4 / 23) \sqrt{69}$. Applying the same proposition to $S=\left\{P_{0}-1, P_{-1}, P_{-2}, \ldots\right\}$ gives

$$
\left|\xi-\frac{18+2 \sqrt{69}}{\varepsilon-1}\right|_{1}=0, \quad \text { with } \quad \frac{18+2 \sqrt{69}}{\varepsilon-1}=\frac{4}{23} \sqrt{69}
$$

hence such $P_{0}=(x, y)$ satisfy $x+y \sqrt{69}=1+(4 / 23) \sqrt{69}$.

Thus any point $\xi=P_{0}$ giving rise to a doubly infinite sequence $\left(P_{j}\right)_{j \in \mathbb{Z}}$ that stays inside $S_{0}$ modulo $\mathcal{O}_{K}$ for all $j \neq 0, \pm 1$ satisfies $\xi=1+(4 / 23) \sqrt{69}$ and $\xi^{\prime}=-(4 / 23) \sqrt{69}$. If we write $P_{0}=(x, y)$, then this gives

$$
x=\frac{1}{2}\left(\xi+\xi^{\prime}\right)=\frac{1}{2} \quad \text { and } \quad y=\frac{1}{2 \sqrt{69}}\left(\xi-\xi^{\prime}\right)=\frac{4}{23}+\frac{1}{2 \sqrt{69}} \approx 0.2341059,
$$

as claimed.

Before we go on exploring the other possibilities, we study the orbit of $P_{0}$, and compute its Euclidean minimum.

Claim 4.3. The points $P_{r} \equiv \varepsilon^{-r} P_{0} \bmod \mathcal{O}_{K}$ in the orbit of $P_{0}$ coincide with the $P_{r}$ given in Theorem 4.1 .

This is done by induction: the case $r=0$ is clear. For the induction step, notice that

$$
\varepsilon^{-1}(x, y)=\left(\frac{25}{2} x-\frac{207}{4} y, \frac{25}{2} y-\frac{3}{2} x\right) ;
$$

now

$$
\begin{aligned}
\varepsilon^{-1} P_{r} & =\left(\frac{25}{4} \varepsilon^{-r}-18-\frac{207}{2 \sqrt{69}} \varepsilon^{-r}, \frac{50}{23}+\frac{25}{4 \sqrt{69}} \varepsilon^{-r}-\frac{3}{4} \varepsilon^{-r}\right) \\
& =(-18,2)+\left(\left(\frac{25}{4}-\frac{3}{4} \sqrt{69}\right) \varepsilon^{-r}, \frac{4}{23}+\left(-\frac{3}{4}+\frac{25}{4 \sqrt{69}}\right) \varepsilon^{-r}\right) \\
& =(-18,2)+\left(\frac{1}{2} \varepsilon^{-r-1}, \frac{4}{23}+\frac{1}{2 \sqrt{69}} \varepsilon^{-r-1}\right) \\
& \equiv P_{r+1} \bmod \mathcal{O}_{K} .
\end{aligned}
$$

Next, one can compute that $\varepsilon P_{0}=(61 / 2,7 / 2)-P_{1}^{\prime}$ and can show, again by induction, that $\varepsilon^{r} P_{0} \equiv-P_{r}^{\prime} \bmod \mathcal{O}_{K}$ for all $r \geqslant 0$. Thus the orbit of $P_{0}$ under the action of the unit group $E_{K}$ of $\mathcal{O}_{K}$ is represented modulo $\mathcal{O}_{K}$ by the points $\left\{ \pm P_{r}, \pm P_{r}^{\prime}: r \geqslant 0\right\}$.

Claim 4.4. The points $P_{r}$ have Euclidean minimum

$$
M\left(K, P_{r}\right)=M\left(K, P_{0}\right)=\frac{15}{46}(11-\sqrt{69}) .
$$




\section{Euclidean windows}

First, we observe that the points $P_{r}$ have the same Euclidean minimum, since they all belong to the same orbit. Now assume that $\varepsilon=t+u \sqrt{m}$ has positive norm. We want to apply Proposition 4.3 and find $\varepsilon^{-1}=t-u \sqrt{m}$; hence

$$
\left(\sqrt{\varepsilon}+\frac{1}{\sqrt{\varepsilon}}\right)^{2}=2 t+2 \text { and } \mu=\sqrt{\frac{k(t+1)}{2}} .
$$

In the case where $m=69$, we have $t=25 / 2$, and hence $\mu / \sqrt{m}=\sqrt{k} \sqrt{27 / 276}<\sqrt{k} / 3$.

The orbit of $P_{0}=1 / 2+(4 / 23+(1 / 2 \sqrt{69})) \sqrt{69}$ is $\left\{ \pm P_{r}, \pm P_{r}^{\prime}: r \in \mathbb{N}_{0}\right\}$, so it is clearly sufficient to compute $M\left(K, P_{r}\right)$ for $r \geqslant 0$. We start with $P_{0}$ itself. The only $\eta \equiv P_{0} \bmod \mathcal{O}_{K}$ satisfying the bounds of Proposition 4.3 have the form $P_{0}+a$ for some $a \in \mathbb{Z}$, or $P_{0}-(b+\sqrt{69}) / 2$ for some odd $b \in \mathbb{Z}$. The minimal absolute value of the norm of these elements is $|N(\eta-(5+\sqrt{69}) / 2)|=(15 / 46)(11-\sqrt{69})$.

Similarly, the minimal norm for the $\eta \equiv P_{1} \bmod \mathcal{O}_{K}$ is attained at $P_{1}+(5-\sqrt{69}) / 2$, and again equals $(15 / 46)(11-\sqrt{69})$.

Finally, consider the $\eta \equiv P_{r} \bmod \mathcal{O}_{K}$ for some $r \geqslant 2$. Then $P_{r}=x_{r}+y_{r} \sqrt{69}$ with $\left|x_{r}\right| \leqslant 0.00081=: \delta_{0}$ and $\left|y_{r}-4 / 23\right|<0.0001=: \delta_{1}$. The minimal absolute value of the norm of $P_{r}+a$ for some $a \in \mathbb{Z}$ is attained for $a=1$, and equals $\left|\left(1+\delta_{0}\right)^{2}-69\left(4 / 23-\delta_{1}\right)^{2}\right| \geqslant$ 1.07; similarly, we find that $\left|N\left(P_{r}-(b+\sqrt{69}) / 2\right)\right| \geqslant 1.07$.

Thus we have seen that inf $\left\{\left|N\left(P_{r}-\alpha\right)\right|: \alpha \in \mathcal{O}_{K}, r \in \mathbb{Z}\right\}$ is attained for $r=0$ and $\alpha=(5+\sqrt{69}) / 2$, giving $M\left(K, P_{0}\right)=(15 / 46)(11-\sqrt{69})$, as claimed.

Before we go on, let us recall what we now know: $K=\mathbb{Q}(\sqrt{69})$ has first minimum $M_{1}(K)=25 / 23$, and $M_{1}$ is isolated. Moreover, the orbit of every $k$-exceptional point for $k=0.875$ that is not congruent to $\pm(4 / 23) \sqrt{69} \bmod \mathcal{O}_{K}$ has a representative in the exceptional set $T$. Finally, if the orbit of such a point visits $T$ exactly once, then the point is $P_{0}=1 / 2+(4 / 23+(1 / 2 \sqrt{69})) \sqrt{69}$, and its minimum is $M\left(K, P_{0}\right)=(15 / 46)(11-\sqrt{69})$.

Claim 4.5. Any exceptional point $Q \neq P_{0}$ in $T$ has Euclidean minimum

$$
M(K, Q)<M\left(K, P_{0}\right)=\frac{15}{46}(11-\sqrt{69})
$$

and $M_{2}(K)=M\left(P_{0}\right)$ is attained only at points in the orbit of $P_{0}$.

In fact, let $Q_{0} \neq P_{0}$ be an exceptional point in $T$, and consider the orbit $\left\{Q_{r}: r \in \mathbb{Z}\right\}$ of $Q_{0}$, where the $Q_{j}$ are defined by $Q_{j} \equiv \varepsilon^{-j} Q_{0} \bmod \mathcal{O}_{K}$. Since $Q_{0} \neq P_{0}$, we know that we are in one of the following situations:

1. (A) and (D) hold;

2. (B) and (C) hold;

3. (B) and (D) hold.

In each case, there exists a point $Q \neq P_{0}$ in $T$, with an orbit that moves into $T$ both to the right and to the left:

$$
\cdots T \longrightarrow S_{2} \longrightarrow S_{0} \cdots S_{0} \longrightarrow S_{1} \longrightarrow Q \longrightarrow S_{2} \longrightarrow S_{0} \cdots S_{0} \longrightarrow S_{1} \longrightarrow T \cdots .
$$

Now we prove the following lemma.

Lemma 4.5. Suppose that there is a $Q_{0} \in T$ such that $Q_{1}=\beta\left(Q_{0}-1\right) \in S_{2}$ and $Q_{m+1}=(x, y)=\beta^{m}\left(Q_{1}\right) \in S_{1}$ with $\beta$ as in map (2). Then $x-y \sqrt{69}<-(4 / 23) \sqrt{69}$. 
Proof. Write $Q_{n}=\left(x_{n}, y_{n}\right)$ and put $\xi_{n}^{\prime}=x_{n}-y_{n} \sqrt{69}$. Then $\xi_{1}^{\prime} \approx-1.48<-(4 / 23) \sqrt{69}$; now we use induction to show that $\xi_{n}^{\prime}<-(4 / 23) \sqrt{69}$ for $1 \leqslant n \leqslant m$.

In fact, if $Q_{n+1}=\beta\left(x_{n}, y_{n}\right)$, then

$$
\begin{aligned}
\xi_{n+1}{ }^{\prime} & =\left(\varepsilon \xi_{n}-(18+2 \sqrt{69})\right)^{\prime} \\
& =\varepsilon^{\prime} \xi_{n}^{\prime}-18+2 \sqrt{69} \\
& <-\varepsilon^{\prime} \frac{4}{23} \sqrt{69}-18+2 \sqrt{69} \\
& =-\frac{4}{23} \sqrt{69} .
\end{aligned}
$$

A similar result holds for the other direction.

Lemma 4.6. Suppose there is a $Q_{0} \in T$ such that $Q_{-1}=\alpha\left(Q_{0}\right) \in S_{1}$ and $Q_{-m-1}=$ $(x, y)=\alpha^{m}\left(Q_{-1}\right) \in S_{2}$. Then $x+y \sqrt{69}>1+(4 / 23) \sqrt{69}$.

Proof. The proof is similar.

This shows that, in orbit (3), we have

$$
\xi>\xi_{0}=1+\frac{4}{23} \sqrt{69} \text { and } \xi^{\prime}<\xi_{0}^{\prime}=-\frac{4}{23} \sqrt{69}
$$

for the point $Q=(x, y)$ and $\xi=x+y \sqrt{69}, \xi^{\prime}=x-y \sqrt{69}$. Put $\alpha=\xi_{0}-(5+\sqrt{69}) / 2$ and $\alpha^{\prime}=\xi_{0}^{\prime}-(5-\sqrt{69}) / 2$. Then $-\alpha \alpha^{\prime}=(15 / 46)(11-\sqrt{69})$, and, since $\alpha<0$ and $\alpha^{\prime}>0,0<(\xi-(5+\sqrt{69}) / 2)\left(\xi^{\prime}-(5-\sqrt{69}) / 2\right)<-\alpha \alpha^{\prime}$. Thus any such point has Euclidean minimum strictly smaller than $(15 / 46)(11-\sqrt{69})$.

Claim 4.6. The second minimum $M_{2}(K)$ is not isolated.

This is accomplished by constructing a series of rational points $Q_{r} \in K \backslash C_{2}$ such that $\lim _{r \rightarrow \infty} M\left(Q_{r}\right)=M_{2}(K)$. To this end, we look for a point $Q_{r} \in T-1$ that gets mapped (multiplication by $\varepsilon$ plus reduction modulo $\mathcal{O}_{K}$ ) to $S_{2}$, stays in $S_{0}$ exactly $r$ times, and then goes to $S_{1}$ and back to the point in $T$ congruent to $Q_{r} \bmod \mathcal{O}_{K}$; then $Q_{r}$ will satisfy the following equation:

$$
\varepsilon^{r+4} Q_{r}=\varepsilon^{r+4}+\left(\varepsilon^{r+3}+\cdots+\varepsilon+1\right)(18+2 \sqrt{69})+Q_{r} .
$$

(For more details, see the analogous construction of the points $R_{r}$ in Section 5.) This gives

$$
Q_{r}=1+\frac{4}{23} \sqrt{69}+\frac{1}{\varepsilon^{r+4}-1} .
$$

Table 1 gives explicit coordinates for small values of $r$.

We claim that $M\left(Q_{r}\right)$ tends to $M_{2}(K)=(15 / 46)(11-\sqrt{69}) \approx 0.87827$ as $r \longrightarrow \infty$. Applying Proposition 4.3 shows that, for given $r \geqslant 0$, the Euclidean minimum of $Q_{r}$ is attained at $Q_{r}-((5+\sqrt{69}) / 2)$. Writing $n=r+4$ and $Q_{r}-((5+\sqrt{69}) / 2)=\left(\xi, \xi^{\prime}\right)$ we have

$$
\begin{aligned}
\xi & =-\frac{3}{2}-\frac{15}{46} \sqrt{69}+\frac{1}{\varepsilon^{n}-1}, \\
\xi^{\prime} & =-\frac{3}{2}+\frac{15}{46} \sqrt{69}+\frac{1}{\varepsilon^{-n}-1}=-\frac{5}{2}+\frac{15}{46} \sqrt{69}-\frac{1}{\varepsilon^{n}-1},
\end{aligned}
$$




\section{Euclidean windows}

Table 1: Euclidean minima of some $Q_{r}$

\begin{tabular}{cccc}
\hline$r$ & $Q_{r}$ & & $M\left(Q_{r}\right)$ \\
\hline-1 & $\frac{1}{2}+\frac{97}{414} \sqrt{69}$ & $\frac{541}{621}$ & $\approx 0.871175523$ \\
0 & $\frac{1}{2}+\frac{70}{299} \sqrt{69}$ & $\frac{13651}{15548}$ & $\approx 0.877990738$ \\
1 & $\frac{1}{2}+\frac{2423}{10350} \sqrt{69}$ & $\frac{340876}{388125}$ & $\approx 0.878263446$ \\
2 & $\frac{1}{2}+\frac{6989}{29854} \sqrt{69}$ & $\frac{8508391}{9687623}$ & $\approx 0.878274371$ \\
3 & $\frac{1}{2}+\frac{30239}{129168} \sqrt{69}$ & $\frac{212369041}{241802496}$ & $\approx 0.878274809$ \\
4 & $\frac{1}{2}+\frac{174445}{745154} \sqrt{69}$ & $\frac{5300717776}{6035374823}$ & $\approx 0.878274826$ \\
\hline
\end{tabular}

and now we find that

$$
\left|N\left(Q_{r}-\frac{5+\sqrt{69}}{2}\right)\right|=-\xi \xi^{\prime}=\frac{165-15 \sqrt{69}}{46}-\frac{1}{\varepsilon^{n}-1}\left(-1+\frac{15}{23} \sqrt{69}\right) .
$$

Since the 'error term'

$$
\frac{1}{\varepsilon^{n}-1}\left(-1+\frac{15}{23} \sqrt{69}\right)
$$

is positive and tends to 0 as $n \longrightarrow \infty$, Claim 4.6 follows, and Theorem 4.1 is proved.

\section{Weighted norms in $\mathbb{Q}(\sqrt{69})$}

Now we study the weighted norm $f_{\mathfrak{p}, c}$ defined by $\mathfrak{p}=(23, \sqrt{69})$. We claim that the following theorem holds.

Theorem 5.1. Let $R=\mathcal{O}_{K}$ be the ring of integers in $K=\mathbb{Q}(\sqrt{69})$, and let $\mathfrak{p}=(23, \sqrt{69})$ be the prime ideal above 23. Then the Euclidean window of $f=f_{\mathfrak{p}, c}$ is $w(\mathfrak{p})=(25, \infty)$; the Euclidean minimum is

$$
M_{1}\left(\mathcal{O}_{K}, f_{\mathfrak{p}, c}\right)=\max \left\{\frac{25}{c}, \frac{75}{23}(-8+\sqrt{69})\right\}
$$

for all $c \in w(\mathfrak{p})$, and $M_{1}$ is isolated exactly when $c \in[23,(23 / 15)(8+\sqrt{69}))$.

Using the method described in [7], with some modifications described in the next section, we can cover the fundamental domain of $\mathcal{O}_{K}$ with a bound of $k=0.99$ except for a set surrounding $(0,0)$ that contains no exceptional point, and $\pm S_{1} \cup \pm S_{2} \cup \pm S_{2}^{\prime}$, where

$$
\begin{array}{rrrrl}
S_{1} & = & {[-0.0084,0.0084]} & \times & {[0.1739,0.175]} \\
S_{2} & = & {[0.2086,0.2087]} & \times & {[0.19903,0.19904]} \\
S_{2}^{\prime} & = & {[0.2086,0.2087]} & \times & {[-0.19904,-0.19903]}
\end{array}
$$




\section{Euclidean windows}

Transforming by units, we find that

$$
\begin{array}{llllll}
\varepsilon S_{1}-(18+2 \sqrt{69}) & \widetilde{\subset} & S_{1} \cup S_{2} ; & \bar{\varepsilon} S_{1}+(18-2 \sqrt{69}) & \widetilde{\subset} & S_{1} \cup\left(-S_{2}^{\prime}\right) ; \\
\varepsilon S_{2}-(23+3 \sqrt{69}) & \widetilde{\subset} & S_{2}^{\prime} ; & \bar{\varepsilon} S_{2}+(18-2 \sqrt{69}) & \widetilde{C} & S_{1} ; \\
\varepsilon S_{2}^{\prime}+(18+2 \sqrt{69}) & \widetilde{\subset} & -S_{1} ; & \bar{\varepsilon} S_{2}^{\prime}-(23-3 \sqrt{69}) & \widetilde{\subset} & S_{2} .
\end{array}
$$

Claim 5.1. If $P$ is an exceptional point that stays inside $S_{1}$ under repeated transformations by $\varepsilon$ and $\varepsilon^{-1}$, then $P=(0,4 / 23)$ has Euclidean minimum $M\left(P, f_{\mathfrak{p}, c}\right)=25 / c$.

This is easy to see. Again, this enables us to reduce everything to exceptional points $P \in S_{2}$, and for the orbit $\left(P_{j}\right)$ of such $P$ (where $P_{j+1}$ is the image of $P_{j}$ under multiplication by $\varepsilon$ plus reduction modulo $\mathcal{O}_{K}$ ) there are the following possibilities.

(a) $P_{j} \in-S_{1}$ and $P_{-j} \in S_{1}$ for all $j \geqslant 2$.

(b) There exist $m \neq n$ such that $P_{m}, P_{n} \in S_{2}$.

Claim 5.2. If $P_{0} \in S_{2}$ is an exceptional point with property (a), then

$$
P_{0}=\left(\frac{-115+15 \sqrt{69}}{46}, \frac{-5+\sqrt{69}}{2 \sqrt{69}}\right) \approx(0.20868169,0.19903536) \text {. }
$$

For a proof, suppose that $P_{0}$ is a point in $S_{2}$ with property (a). Then

$$
P_{1}=-\varepsilon P_{0}+(23+3 \sqrt{69}) \in-S_{2}^{\prime},
$$

and $P_{2}=\varepsilon P_{1}-(18+2 \sqrt{69})$ is a point for which the transforms by powers of $\varepsilon$ stay inside $S_{1}$. By Proposition 4.2, this implies that $\left|P_{2}-(4 / 23) \sqrt{69}\right|_{1}=0$, and going back to $P_{0}$ we find that $\left|P_{0}-(-5+(19 / 23) \sqrt{69})\right|_{1}=0$.

Similarly, any exceptional point $\xi \in S_{2}$ with transforms by powers of $\bar{\varepsilon}$ that stay inside $S_{1}$ satisfies $|\xi+(4 / 23) \sqrt{69}|_{2}=0$. Thus any point satisfying property (a) has $x$-coordinate

$$
\frac{\xi+\xi^{\prime}}{2}=\frac{-115+15 \sqrt{69}}{46}
$$

and $y$-coordinate

$$
\frac{\xi-\xi^{\prime}}{2 \sqrt{69}}=\frac{-5+\sqrt{69}}{2 \sqrt{69}}
$$

as claimed.

Note that there is no obvious definition of a 'Euclidean minimum' of $P_{0}$ with respect to weighted norms $f_{\mathfrak{p}, c}$, since $f_{\mathfrak{p}, c}$ is a continuous function on $K$ (with respect to the topology inherited from the embedding $K \longrightarrow \mathbb{R}^{2}$ ) if and only if $c=\mathfrak{p}$; that is, if and only if $f_{\mathfrak{p}, c}$ is the absolute value of the usual norm. Thus we cannot extend $f_{\mathfrak{p}, c}$ by continuity to $\mathbb{R}^{2}$. On the other hand, we can put

$$
\bar{M}\left(P, f_{\mathfrak{p}, c}\right)=\sup \left\{M\left(P_{r}, f_{\mathfrak{p}, c}\right): P_{r} \in K, \lim P_{r}=P\right\} ;
$$

that is, we define the minimum at a point $P \in K$ as the supremum of the minima at $P_{r} \in K$ over all sequences $\left(P_{r}\right)$ converging to $P$ in the topology mentioned above. If $P \in K$, then clearly $\bar{M}\left(P, f_{\mathfrak{p}, c}\right) \geqslant M\left(P, f_{\mathfrak{p}, c}\right)$, as the constant series $P_{r}=P$ shows. We do not know of any example where this last inequality is strict. 
Claim 5.3. We have $\bar{M}\left(P_{0}\right) \leqslant \kappa_{0}=(75 / 23)(-8+\sqrt{69})$ for all $c \geqslant 23$. Moreover, any $K$-rational exceptional point with property (b) has minimum strictly smaller than $\kappa_{0}$. In particular, we have $M_{1}(K)=25 / c$ for all $c \in[23,(25 / 23)(24+3 \sqrt{69})]$, and $M_{1}$ is isolated for these values of $c$, except possibly when $c=(25 / 23)(24+3 \sqrt{69})$.

We start by observing that

$$
\begin{aligned}
\left|N\left(P_{0}-2\right)\right| & =\frac{94-10 \sqrt{69}}{23} \approx 0.47538092916, \quad \text { and } \\
\left|N\left(P_{0}-\frac{1}{2}(5+\sqrt{69})\right)\right| & =\frac{-600+75 \sqrt{69}}{23} \approx 0.99986042255 .
\end{aligned}
$$

Using the same technique as in Lemmas 4.5 and 4.6, we can show that the $K$-rational points in $S_{2}$ that satisfy condition (b) have a minimum that is strictly smaller than $\kappa_{0}$; observe that the difference $\eta_{1}-\eta_{2}$ for $\eta_{1}=(5+\sqrt{69}) / 2$ and $\eta_{2}=2$ is not divisible by $\mathfrak{p}$, and hence we have $f_{\mathfrak{p}, c}\left(P_{0}-\eta_{j}\right) \leqslant\left|N\left(P_{0}-\eta_{j}\right)\right|$ for $j=1$ or $j=2$. Since any sequence of $K$-rational points $P_{r}$ converging to $P_{0}$ eventually stays inside $S_{2}$ this also proves that $M_{1}\left(\mathcal{O}_{K}, f_{\mathfrak{p}, c}\right)=25 / c$ as long as $25 / c \geqslant \kappa_{0}$; but the last inequality holds for all $c \leqslant(23 / 15)(8+\sqrt{69}) \approx 25.0034899$. It also shows that the minimum is isolated for these values, except possibly when $c=(23 / 15)(8+\sqrt{69})$.

Claim 5.4. We have $\bar{M}\left(P_{0}, f_{\mathfrak{p}, c}\right)=\kappa_{0}=(75 / 23)(-8+\sqrt{69})$ for all $c>23$, and $\bar{M}\left(P_{0}, f_{\mathfrak{p}, c}\right)=M(P)=(94-10 \sqrt{69}) / 23$ for $c=23$.

In order to show that $\kappa_{0}$ is a lower bound for $M\left(P_{0}\right)$ for $c>23$, we construct a series of $K$-rational points converging to $P_{0}$, with minima that converge to $\kappa_{0}$. We do this in the following way: assume that $R_{r} \in S_{2}$ gets mapped to $S_{2}^{\prime}$, stays in $-S_{1}$ exactly $r-2$ times and then gets mapped to the point $-R_{r} \in-S_{2}$. Then

$$
\begin{aligned}
& \varepsilon R_{r}-(23+3 \sqrt{69}) \in S_{2}^{\prime}, \\
& \varepsilon^{2} R_{r}-\varepsilon(23+3 \sqrt{69})+(18+2 \sqrt{69}) \in-S_{1}, \ldots, \\
& \varepsilon^{r} R_{r}-\varepsilon^{r-1}(23+3 \sqrt{69})+(18+2 \sqrt{69})\left(1+\varepsilon+\cdots+\varepsilon^{r-2}\right) \in-S_{1}
\end{aligned}
$$

and finally

$$
\left(\varepsilon^{r+1}+1\right) R_{r}=\varepsilon^{r}(23+3 \sqrt{69})-(18+2 \sqrt{69}) \frac{\varepsilon^{r}-1}{\varepsilon-1} .
$$

Now we use $\left(\varepsilon^{r}-1\right) /(\varepsilon-1)=\left(\varepsilon^{r+1}-1\right) /(\varepsilon-1)-\varepsilon^{r}$ to find

$$
\begin{aligned}
\left(\varepsilon^{r+1}+1\right) R_{r} & =\varepsilon^{r}(41+5 \sqrt{69})-(18+2 \sqrt{69}) \frac{\varepsilon^{r+1}-1}{\varepsilon-1} \\
& =\varepsilon^{r+1}(-5+\sqrt{69})-(18+2 \sqrt{69}) \frac{\varepsilon^{r+1}-1}{\varepsilon-1} .
\end{aligned}
$$

Dividing through by $\varepsilon^{r+1}+1$ and simplifying, we get

$$
R_{r}=-5+\frac{19}{23} \sqrt{69}+\frac{1}{\varepsilon^{r+1}+1}\left(5-\frac{15}{23} \sqrt{69}\right) .
$$

The explicit coordinates for the first few points are given in Table 2.

Claim 5.5. The Euclidean minimum of $R_{r}(r \geqslant 2)$ with respect to $f_{\mathfrak{p}, c}$ is attained at $R_{r}-2$ or $R_{r}-(5+\sqrt{69}) / 2$. 


\section{Euclidean windows}

Table 2: Euclidean minima of some $R_{r}$

\begin{tabular}{cccccc}
\hline$r$ & $R_{r}$ & $\left|N\left(R_{r}-\frac{1}{2}(5+\sqrt{69})\right)\right|$ & $\left|N\left(R_{r}-2\right)\right|$ \\
\hline 1 & $\frac{1}{5}+\frac{1}{5} \sqrt{69}$ & $\frac{23}{25}$ & $=0.92$ & $\frac{12}{25}$ & $=0.48$ \\
2 & $\frac{5}{24}+\frac{43}{216} \sqrt{69}$ & $\frac{3875}{3888}$ & $\approx 0.996656378$ & $\frac{1849}{3888}$ & $\approx 0.475565843$ \\
3 & $\frac{130}{623}+\frac{124}{623} \sqrt{69}$ & $\frac{388025}{388129}$ & $\approx 0.999732047$ & $\frac{184512}{388129}$ & $\approx 0.475388337$ \\
4 & $\frac{125}{599}+\frac{1073}{5391} \sqrt{69}$ & $\frac{9686225}{9687627}$ & $\approx 0.999855279$ & $\frac{4605316}{9687627}$ & $\approx 0.475381225$ \\
5 & $\frac{649}{3110}+\frac{619}{3110} \sqrt{69}$ & $\frac{2417687}{2418025}$ & $\approx 0.999860216$ & $\frac{1149483}{2418025}$ & $\approx 0.475380941$ \\
6 & $\frac{3120}{14951}+\frac{26782}{134559} \sqrt{69}$ & $\frac{6034532375}{6035374827}$ & $\approx 0.999860414$ & $\frac{2935561516}{6035374827}$ & $\approx 0.475380929$ \\
\hline
\end{tabular}

In fact, by applying Proposition 4.3 to $R_{r}$, one checks that the two smallest values of $\left|N\left(R_{r}-\eta\right)\right|$ occur for $\eta_{1}=2$ or $\eta_{2}=(5+\sqrt{69}) / 2$; one also verifies that $\left|N\left(R_{r}-2\right)\right| \approx$ 0.47 and $\left|N\left(R_{r}-(5+\sqrt{69}) / 2\right)\right| \approx 0.99$. Since the denominator of $R_{r}-\eta$ is not divisible by $\mathfrak{p}$ for any $\eta \in \mathcal{O}_{K}$ (it divides $\varepsilon^{r+1}+1 \equiv 2 \bmod \mathfrak{p}$ ), and since $\eta_{1}-\eta_{2}$ is an integer not divisible by $\mathfrak{p}$, our claim follows.

Where the minimum with respect to $f_{\mathfrak{p}, c}$ is attained depends on whether the numerator of $R_{r}-2$ is divisible by $\mathfrak{p}$ or not: if it is not, then the Euclidean minimum is attained there, and we have $M\left(P, f_{\mathfrak{p}, c}\right)=\left|N\left(R_{r}-2\right)\right|<1 / 2$. If this numerator, however, $i s$ divisible by $\mathfrak{p}$, then $f_{\mathfrak{p}, c}\left(R_{r}-2\right)$ can be made as large as we please by adding weight to $\mathfrak{p}$, and in this case the minimum is attained at $R_{r}-(5+\sqrt{69}) / 2$ for large values of $c$.

Claim 5.6. The numerator of $R_{r}-2$ is divisible by $\mathfrak{p}$ if and only if $r \equiv 10 \bmod 23$. In this case, it is even divisible by $(23)=\mathfrak{p}^{2}$.

Let us compute $R_{r} \bmod \mathfrak{p}$. Since $\varepsilon \equiv 1 \bmod \mathfrak{p}$, we find that

$$
\frac{\varepsilon^{r+1}-1}{\varepsilon-1}=1+\varepsilon+\cdots+\varepsilon^{r} \equiv r+1 \bmod \mathfrak{p} ;
$$

hence

$$
2 R_{r}=\varepsilon^{r}(23+3 \sqrt{69})-(18+2 \sqrt{69}) \frac{\varepsilon^{r}-1}{\varepsilon-1} \equiv 5 r \bmod \mathfrak{p},
$$

and therefore $R_{r}-2 \equiv 0 \bmod \mathfrak{p}$ if and only if $5 r \equiv 4 \bmod 23$, which in turn is equivalent to $r \equiv 10 \bmod 23$.

The second part of the claim follows by observing that

$$
\varepsilon^{s} \equiv(1+13 \sqrt{69}) \equiv 1+13 s \sqrt{69} \bmod 23 ;
$$

in particular,

$$
\varepsilon^{23 m+10} \equiv 1+13 \sqrt{69} \bmod 23
$$

and

$$
\frac{\varepsilon^{r}-1}{\varepsilon-1}=\varepsilon^{r-1}+\cdots+\varepsilon+1 \equiv r+1+13 \frac{r(r+1)}{2} \sqrt{69} \bmod 23 .
$$


With a little more effort, we can show much more, namely that there is a subsequence of $R_{r}-2$ with numerators divisible by an arbitrarily large power of $\mathfrak{p}$. In fact, the numerator of $R_{r}-2$ will be divisible by $\mathfrak{p}^{k}$ if and only if $T_{r}=23\left(\varepsilon^{r+1}+1\right)\left(R_{r}-2\right) \equiv 0 \bmod \mathfrak{p}^{k+2}$, and here $T_{r}$ is an algebraic integer. An elementary calculation shows that the last congruence is equivalent to

$$
\varepsilon^{r+1} \equiv-\frac{47+5 \sqrt{69}}{22}=: \alpha \bmod \mathfrak{p}^{k+2} .
$$

This will hold for arbitrarily large $k$ if and only if there is a 23-adic integer $s=r+1$ such that

$$
\varepsilon^{s}=\alpha
$$

holds in $K_{\mathfrak{p}}=\mathbb{Q}_{23}(\sqrt{69})$. Since both sides are congruent $1 \bmod \mathfrak{p}$, we can take the $\pi$-adic $\operatorname{logarithm}$ (with $\pi=(23+3 \sqrt{69}) / 2$ ) and get $s=\left(\log _{\pi} \alpha\right) /\left(\log _{\pi} \varepsilon\right)$ as an equation in $K_{\mathfrak{p}}$, and equation (5) holds if we can show that $s$ is in $\mathbb{Z}_{23}$. To this end, let $\sigma$ denote the non-trivial automorphism of $K_{\mathfrak{p}} / \mathbb{Q}_{23}$. Since $\log _{\pi}$ is Galois-equivariant, and since $\varepsilon^{1+\sigma}=\alpha^{1+\sigma}=1$, we get

$$
s^{\sigma}=\frac{\log _{\pi} \alpha^{\sigma}}{\log _{\pi} \varepsilon^{\sigma}}=\frac{-\log _{\pi} \alpha}{-\log _{\pi} \varepsilon}=s .
$$

Thus $s \in \mathbb{Q}_{23}$, and since it is a $\pi$-adic unit, $s \in \mathbb{Z}_{23}$ as desired. We remark that $s=11+13 \cdot 23+15 \cdot 23^{2}+5 \cdot 23^{3}+3 \cdot 23^{4}+\cdots$.

This proves Claim 5.4, and completes the proof of Theorem 5.1.

\section{Weighted norms in cubic number fields}

Using the idea of Clark (see $[7, \mathbf{8}, \mathbf{1 1}, \mathbf{1 7}]$; it actually first appeared in work by Lenstra $[14$, p. 35]), we slightly modified the programs described in [6] in order to examine weighted norms in cubic fields. Many of the results in this section were obtained by the first author, and described in [5]; see Table 3 for the results obtained so far.

The idea is simple. Assume that $K$ is a number field with class number 1 , such that $M=M_{1}(K) \geqslant 1$ and $M_{2}(K)<1$; assume that $\# C_{1}(K)$ is finite, and write the points $\xi \in C_{1}(K)(1 \leqslant i \leqslant t)$ in the form $\xi_{i}=\alpha_{i} / \beta_{i}$, where $\left(\alpha_{i}, \beta_{i}\right)=1$. Assume, moreover, that there is a prime ideal $\mathfrak{p}$ such that $\mathfrak{p} \mid \beta_{i}$ for all $i$.

Now consider the weighted norm $f_{\mathfrak{p}, c}$; by making $c$ big enough we can certainly arrange that $f_{\mathfrak{p}, c}\left(\xi_{i}\right)<1$ for all $i \leqslant t$; in fact, if $\mathfrak{p}^{m} \| \operatorname{gcd}\left(\beta_{1}, \ldots, \beta_{t}\right)$, then $f_{\mathfrak{p}, c}\left(\xi_{i}\right) \leqslant M(N \mathfrak{p})^{m} c^{-m}$; thus we need only to choose $c>N \mathfrak{p} \sqrt[m]{M}$. (Actually, this shows that $w(\mathfrak{p}) \subseteq(N \mathfrak{p} \sqrt[m]{M}, \infty)$.)

To guarantee that, for every $\xi \in K$, there exists a $\gamma \in \mathcal{O}_{K}$ such that $f_{\mathfrak{p}, c}(\xi-\gamma)<1$, we shall look for $\gamma_{1}, \gamma_{2} \in \mathcal{O}_{K}$ such that $\left|N_{K / \mathbb{Q}}\left(\xi-\gamma_{i}\right)\right|<1$ for $i=1,2$ and $\mathfrak{p} \nmid\left(\gamma_{1}-\gamma_{2}\right)$; then at least one of the $\xi-\gamma_{i}$, say $\xi-\gamma_{1}$, has a numerator that is not divisible by $\mathfrak{p}$, and this implies that $f_{\mathfrak{p}, c}\left(\xi-\gamma_{1}\right) \leqslant\left|N\left(\xi-\gamma_{1}\right)\right|<1$.

Modifications of the programs described in [6] allow us to find new examples of cubic fields that are not norm-Euclidean, but are Euclidean with respect to some weighted norm. We represented prime ideals of the maximal order $\mathcal{O}_{K}=\mathbb{Z} \oplus \alpha \mathbb{Z} \oplus \beta \mathbb{Z}$ in the form $\mathfrak{p}=(p, \alpha+a), \mathfrak{p}=(p, \beta+a \alpha+b)$ or $\mathfrak{p}=(p)$, according to whether $\mathfrak{p}$ has degree 1,2 or 3 . Testing the divisibility of an integer of $\mathcal{O}_{K}$ by $\mathfrak{p}$ can then be done using only rational arithmetic. 


\section{Euclidean windows}

Table 3: Euclidean windows for cubic fields

\begin{tabular}{|c|c|c|c|c|}
\hline $\operatorname{disc} K$ & $M_{1}(K)$ & $M_{2}(K)$ & $N \mathfrak{p}$ & $\mathrm{w}(\mathfrak{p})$ \\
\hline-367 & 1 & $9 / 13$ & 13 & $(13,279 / 8)$ \\
\hline-351 & 1 & $9 / 11$ & 11 & $(11, \infty)$ \\
\hline-327 & $101 / 99$ & $<0.9$ & 11 & $(101 / 9, \infty)$ \\
\hline-199 & 1 & $<0.47$ & 7 & $(7, \infty)$ \\
\hline 985 & 1 & $5 / 11$ & 5 & $(5, \infty)$ \\
\hline 1345 & $7 / 5$ & $<0.4$ & 5 & $(7, \infty)$ \\
\hline 1825 & $7 / 5$ & $<0.5$ & 5 & $(7, \infty)$ \\
\hline 1929 & 1 & $3 / 7$ & 7 & $(7, \infty)$ \\
\hline 1937 & 1 & $5 / 9$ & 3 & $(3, \infty)$ \\
\hline 2777 & $5 / 3$ & $17 / 19$ & 3 & $\varnothing$ \\
\hline 2836 & $7 / 4$ & $7 / 8$ & 2 & $(\sqrt{7}, \infty)$ \\
\hline 2857 & $8 / 5$ & $<0.5$ & 5 & $(8, \infty)$ \\
\hline 3305 & $13 / 9$ & $37 / 45$ & 3 & $(\sqrt{13}, 5)$ \\
\hline 3889 & $13 / 7$ & 1 & 7 & $(13, \infty)$ \\
\hline 4193 & $7 / 5$ & $<0.65$ & 5 & $(7, \infty)$ \\
\hline 4345 & $7 / 5$ & $11 / 13$ & 5 & $(7, \infty)$ \\
\hline 4360 & $41 / 35$ & $7 / 10$ & 7 & $(41 / 5, \infty)$ \\
\hline 5089 & $17 / 11$ & $7 / 11$ & 11 & $(17, \infty)$ \\
\hline 5281 & 1 & $<0.6$ & 5 & $(5, \infty)$ \\
\hline 5297 & $21 / 11$ & $23 / 33$ & 11 & $(21, \infty)$ \\
\hline 5329 & $9 / 8$ & $63 / 73$ & $2^{3}$ & $(9,73)$ \\
\hline 5369 & $21 / 19$ & $17 / 19$ & 19 & $(21, \infty)$ \\
\hline 5521 & $23 / 7$ & $8 / 7$ & 7 & $(23, \infty)$ \\
\hline 7273 & $973 / 601$ & $729 / 601$ & 601 & $(973, \infty)$ \\
\hline 7465 & 1 & $<0.8$ & 5 & $(5, \infty)$ \\
\hline 7481 & 1 & $<0.7$ & 5 & $(5, \infty)$ \\
\hline
\end{tabular}




\section{Euclidean windows}

Let us call $\xi \in K$ covered if there exist $\gamma_{1}, \gamma_{2} \in \mathcal{O}_{K}$ such that $\left|N_{K / \mathbb{Q}}\left(\xi-\gamma_{i}\right)\right|<1$ and $\mathfrak{p} \nmid\left(\gamma_{1}-\gamma_{2}\right)$; if $\xi$ is covered, then so is $\varepsilon \xi$ for any unit $\varepsilon \in \mathcal{O}_{K}^{\times}$(this allows us to use the program E-3 of [6]).

We first consider the field $K$ generated by a root $\alpha$ of $x^{3}+x^{2}-6 x-1$; we have $\operatorname{disc} K=985$, and the only point with minimum greater than or equal to 1 is

$$
\xi_{1}=\frac{3 \alpha-\alpha^{2}}{\alpha-1}=\frac{2-\alpha+2 \alpha^{2}}{5} .
$$

The ideal $\mathfrak{p}=(\alpha-1)$ occurring in the denominator is a prime ideal of norm 5. Our programs cover a fundamental domain of $K$, except for the possible exceptional points $\xi=0$ and $\xi=\xi_{1}$. Thus $f_{\mathfrak{p}, c}$ is a Euclidean function for every $c>N \mathfrak{p}=5$; that is, $w(\mathfrak{p})=(5, \infty)$.

Now let $K$ be the field with disc $K=1937$ generated by a root $\alpha$ of $x^{3}+x^{2}-8 x+1$. It has Euclidean minimum $M(K)=1$ attained at $\left(4+4 \alpha^{2}\right) / 9$; in fact, $\left|N\left(\xi_{1}\right)\right|=1$ for $\xi_{1}=\left(-14+9 \alpha+4 \alpha^{2}\right) / 9$, and the prime ideal factorization of $\xi_{1}$ is

$$
\left(\xi_{1}\right)=\left(3, \alpha^{2}+1\right)(3, \alpha+1)^{-2} .
$$

Our programs cover a fundamental domain of $K$, except for the possible exceptional points $\xi_{0}=0, \xi=\xi_{1}$ and $\xi=\left(1+\alpha^{2}\right) / 3$. This last point has Euclidean minimum $1 / 3=\left|N\left(1-3 \alpha+\alpha^{2}\right) / 3\right|$ with respect to the usual norm, and since $\left(1-3 \alpha+\alpha^{2}\right) / 3=\mathfrak{p}^{-1}$, adding weight to $\mathfrak{p}$ does not increase its minimum.

Our third example is the cubic field $K$ with discriminant disc $K=3305$, generated by a root $\alpha$ of $x^{3}-x^{2}-10 x-3$. It has minimum $M_{1}=13 / 9$, attained at $\left(1-2 \alpha-4 \alpha^{2}\right) / 9$, with $\left|N\left(\xi_{1}\right)\right|=13 / 9$ for $\xi_{1}=\left(-71+52 \alpha+32 \alpha^{2}\right) / 9$. Its prime ideal factorization is $\left(\xi_{1}\right)=(13, \alpha-1)(3, \alpha)^{-2}$; we thus add weight $c>\sqrt{13}$ to $\mathfrak{p}=(3, \alpha)$, and we can cover a fundamental domain of $K$, except for the possible exceptional points $\xi_{0}=0, \xi=\xi_{1}$ and $\xi=\left(2-\alpha+2 \alpha^{2}\right) / 5$. Now $M(\xi)=\left|N\left(\xi_{2}\right)\right|=3 / 5$, where $\xi_{2}=\left(-3+4 \alpha+2 \alpha^{2}\right) / 5$ has the prime ideal factorization $\left(\xi_{2}\right)=\mathfrak{p}(5, \alpha+2)^{-1}$. Thus the weighted prime ideal occurs in the numerator of $\xi_{2}$, and we have $f_{\mathfrak{p}, c}\left(\xi_{2}\right)<1$ if and only if $c<5$; since $|N(\xi)| \geqslant 1$ for all $\xi \equiv \xi_{2} \bmod \mathcal{O}_{K}$, this implies that $w(\mathfrak{p})=(\sqrt{13}, 5)$.

Finally, consider the cubic field $K$ with discriminant disc $K=3889$. Its first minimum is attained at $\xi_{1}=\left(3-\alpha-3 \alpha^{2}\right) / 7$, and its denominator is the prime ideal $\mathfrak{p}$ that divides the denominator of $\xi_{2}=\left(2-3 \alpha-2 \alpha^{2}\right) / 7$, where the second minimum $M_{2}(K)=1$ is attained. (Something similar happens for $\operatorname{disc} K=5521$ and $\operatorname{disc} K=7273$, where $M_{2}(K)>1$; in these cases, we have to verify that $M_{3}(K)<1$.) Here we find the possible exceptional points $\xi=0, \xi_{1}$, and $\xi_{2}$, as well as

$$
\eta_{1}=\frac{1}{7}\left(1-\alpha-2 \alpha^{2}\right), \quad \eta_{2}=\frac{1}{7}\left(2-2 \alpha+3 \alpha^{2}\right) \quad \text { and } \quad \eta_{3}=\frac{1}{7}\left(3-3 \alpha+\alpha^{2}\right) .
$$

Since their denominator is the prime ideal $(7,2+\alpha)$, their Euclidean minimum is $1 / 7$, for both the usual and the weighted norm.

Some of our examples of cubic fields that are Euclidean with respect to some weighted norm were found independently by Amin Coja-Oghlan, and are described in his thesis [10].

\section{Norm-Euclidean cubic fields}

We take this opportunity to report on recent computations concerning norm-Euclidean cubic fields. Calculations for the totally real cubic fields up to disc $K \leqslant 13,000$ have produced the results shown in Table 4 


\section{Euclidean windows}

Table 4: Norm-Euclidean real cubic fields

\begin{tabular}{crrr}
\hline disc $K$ & $\mathrm{E}$ & $\mathrm{N}$ & $\Sigma$ \\
\hline $0<d \leqslant 1000$ & 26 & 1 & 27 \\
$1000<d \leqslant 2000$ & 29 & 5 & 34 \\
$2000<d \leqslant 3000$ & 31 & 4 & 35 \\
$3000<d \leqslant 4000$ & 36 & 6 & 42 \\
$4000<d \leqslant 5000$ & 28 & 7 & 35 \\
$5000<d \leqslant 6000$ & 35 & 7 & 42 \\
$6000<d \leqslant 7000$ & 30 & 8 & 38 \\
$7000<d \leqslant 8000$ & 37 & 10 & 47 \\
$8000<d \leqslant 9000$ & 30 & 11 & 41 \\
$9000<d \leqslant 10000$ & 29 & 10 & 39 \\
$10000<d \leqslant 11000$ & 34 & 9 & 43 \\
$11000<d \leqslant 12000$ & 37 & 16 & 53 \\
$12000<d \leqslant 13000$ & 31 & 6 & 37 \\
\hline$\Sigma$ & 413 & 100 & 513
\end{tabular}

The columns $E$ and $N$ display the number of norm-Euclidean and non-norm-Euclidean number fields of fields with discriminants in the indicated intervals.

We also have to correct the entries for the fields with discriminant 3969 in our tables in [6]: the field $K_{1}$ generated by a root of $x^{3}-21 x-28$ has $M_{1}\left(K_{1}\right)=4 / 3, M_{2}\left(K_{1}\right)=31 / 24$ and $M_{3}\left(K_{1}\right)=1$, and the field $K_{2}$ generated by $x^{3}-21 x-35$ has $M_{1}\left(K_{2}\right)=7 / 3$ and $M_{2}\left(K_{2}\right)=125 / 63$.

For complex cubic fields, calculations by R. Quême have indicated that the fields with discriminants disc $K=-999$ and disc $K=-1055$ are not norm-Euclidean, and we have subsequently been able to verify that $M(K) \geqslant 294557 / 272112$ for disc $K=-999$, that $M(K) \geqslant 1483 / 1370$ for disc $K=-1055$, and that there are no norm-Euclidean number fields with $-876>$ disc $K \geqslant-1600$, suggesting the following conjecture.

Conjecture. There are exactly 58 norm-Euclidean complex cubic fields, and their discriminants are: $-23,-31,-44,-59,-76,-83,-87,-104,-107,-108,-116,-135,-139$, $-140,-152,-172,-175,-200,-204,-211,-212,-216,-231,-239,-243,-244$, $-247,-255,-268,-300,-324,-356,-379,-411,-419,-424,-431,-440,-451$, $-460,-472,-484,-492,-499,-503,-515,-516,-519,-543,-628,-652,-687$, $-696,-728,-744,-771,-815,-876$.

Note that, by a result of Cassels [4], there are only finitely many norm-Euclidean complex cubic number fields $K$, and in fact their discriminant is bounded by $\mid$ disc $K \mid<170520$.

In the real case, the situation is not so clear. The numerical data suggest that the proportion of norm-Euclidean fields is decreasing with disc $K$, but they do not yet support the conjecture that the norm-Euclidean real cubic number fields have density 0 among the real cubic fields 
Table 5: Norm-Euclidean complex cubic fields

\begin{tabular}{crrr}
\hline$d=|\operatorname{disc} K|$ & $\mathrm{E}$ & $\mathrm{N}$ & $\Sigma$ \\
\hline $0<d \leqslant 200$ & 18 & 1 & 19 \\
$200<d \leqslant 400$ & 15 & 9 & 24 \\
$400<d \leqslant 600$ & 16 & 10 & 26 \\
$600<d \leqslant 800$ & 7 & 20 & 27 \\
$800<d \leqslant 1000$ & 2 & 29 & 31 \\
$1000<d \leqslant 1200$ & 0 & 29 & 29 \\
$1200<d \leqslant 1400$ & 0 & 35 & 35 \\
$1400<d \leqslant 1600$ & 0 & 27 & 27 \\
\hline$\Sigma$ & 58 & 160 & 218 \\
\hline
\end{tabular}

with class number 1 ; in particular, there may be infinitely many norm-Euclidean real cubic number fields.

\section{Some open problems}

In this last section we should like to mention several open problems concerning the Euclidean algorithm with respect to weighted norms. One of the most widely studied questions is, of course, whether $\mathbb{Z}[\sqrt{14}]$ is Euclidean with respect to some $f_{\mathfrak{p}, c}$, where $\mathfrak{p}=(2, \sqrt{14})$. Is it true, in particular, that $w(\mathfrak{p})=(\sqrt{5}, \sqrt{7})$ in this case?

More generally, assume that $K$ is a number field with unit rank $\geqslant 1$. Is $w(\mathfrak{p})$ always an open subset of $(1, \infty) \subset \mathbb{R}$ for every prime ideal $\mathfrak{p}$ in $\mathcal{O}_{K}$ ? If this were the case, then there would also exist number fields such that $f_{\mathfrak{p}, c}$ is a Euclidean function for some $c<N \mathfrak{p}$, since there do exist number fields with $w(\mathfrak{p}) \supseteq[p, \infty)$ for suitable primes (take norm-Euclidean fields, for example).

A related question is whether $M\left(f_{\mathfrak{p}, c}\right)$ is a continuous function of $c$ on $[N \mathfrak{p}, \infty)$ for number fields with unit rank $\geqslant 1$.

The cubic field with discriminant disc $K=-335$ has $M_{1}(K)=1$; the minimum is attained at points that have different prime ideals above 5 in their denominators. Calculations have not yet confirmed that $\mathcal{O}_{K}$ is Euclidean with respect to a norm that is weighted at two different prime ideals. Similar remarks apply to algorithms with respect to functions that are not multiplicative: instead of giving weight $c$ to a prime ideal $\mathfrak{p}$, one could look at functions with $f(\mathfrak{p})=N \mathfrak{p}$ and $f\left(\mathfrak{p}^{2}\right)=c$ for some $c \geqslant N \mathfrak{p}^{2}$. This idea is applicable whenever the denominators of the exceptional points are divisible by the square of a prime ideal; for example, for $\mathbb{Z}[\sqrt{14}]$.

Acknowledgements. We thank-in chronological order-Hendrik Lenstra, Gerhard Niklasch and David Kohel for the argument used to prove Claim 5.4.

Note added in proof. Malcolm Harper [12] has proved that, in particular, the ring $\mathbb{Z}[\sqrt{14}]$ is Euclidean with respect to a suitable Euclidean function, by extending the methods used in [9]. The question of whether $\mathbb{Z}[\sqrt{14}]$ is Euclidean with respect to a weighted norm remains open. 


\section{References}

1. E. S. BARNES and H.P. F. SWINNERTON-DYER, 'The inhomogeneous minima of binary quadratic forms I', Acta Math. 87 (1952), 259-323; II, ibid., 88 (1952) 279-316. 340

2. E. Bedocchi, 'L'anneau $\mathbb{Z}[\sqrt{14}]$ et l'algorithme Euclidien', Manuscripta Math. 53 (1985) 199-216. 338

3. D. CARdon, 'A Euclidean ring containing $\mathbb{Z}[\sqrt{14}]$ ', C. R. Math. Rep. Acad. Sci. Canada 19 (1997) 28-32. 338

4. J. W. S. CASSELS, 'The inhomogeneous minima of binary quadratic, ternary cubic, and quaternary quartic forms', Proc. Cambridge Phil. Soc. 48 (1952) 72-86; Addendum, ibid., 519-520. 352

5. S. Cavallar, 'Alcuni esempi di campi di numeri cubici non euclidei rispetto alla norma ma euclidei rispetto ad una norma pesata', Tesi di Laurea, Universita di Trento, 1995. 349

6. S. Cavallar and F. Lemmermeyer, 'The Euclidean algorithm in cubic number fields', Proceedings, Number Theory, Eger 1996 (ed. K. Györy, A. Pethö and V. T. Sos, Gruyter, 1998) 123-146. 336, 340, 340, 349, 349, 351, 352

7. D. A. Clark, 'A quadratic field which is Euclidean but not norm-Euclidean', Manuscripta Math. 83 (1994) 327-330. 337, 338, 345, 349

8. D. A. Clark, 'Non-Galois cubic fields which are Euclidean but not norm-Euclidean', Math. Comp. 65 (1996) 1675-1679. 337, 349

9. D. A. Clark and R. Murty, 'The Euclidean algorithm for Galois extensions of $\mathbb{Q}$ ', J. Reine Angew. Math. 459 (1995) 151-162. 353

10. A. Coja-Oghlan, 'Berechnung kubischer euklidischer Zahlkörper', Diplomarbeit, FU Berlin, 1999. 351

11. B. Hainke, 'Euklidische Algorithmen in den Ganzheitsringen von $\mathbb{Q}(\sqrt{14})$ und $\mathbb{Q}(\sqrt{69})$ ', Diplomarbeit, Universität Mainz, 1998. 338, 349

12. M. Harper, 'A proof that $\mathbb{Z}[\sqrt{14}]$ is a Euclidean domain', Ph.D. thesis, McGill University, 2000; http://euclid.math.mcgill.ca/harper/. 353

13. F. LeMmermeyer, 'The Euclidean algorithm in algebraic number fields', Expo. Math. 13 (1995) 385-416. 336, 337, 339, 339

14. H. W. Lenstra, Euclidean rings, Lecture Notes, Bielefeld (University of Bielefeld, 1974). 337, 337, 349

15. M. NAGATA, 'A pairwise algorithm and its application to $\mathbb{Z}[\sqrt{14}]$ ', Proc. Algebraic Geometry Seminar, Singapore 1987 (World Scientific Publishing, Singapore, 1988) 69-74. 338

16. M. NAGATA, 'Some questions on $\mathbb{Z}[\sqrt{14}]$ ', Algebraic geometry and its applications, Proc. Conf., Purdue University, USA, June 1-4, 1990 (ed. Ch. Bajaj, Springer, 1994) 327-332. 338

17. G. NiKLASCH, 'On Clark's example of a Euclidean field which is not norm-euclidean', Manuscripta Math. 83 (1994) 443-446. 338, 349 
Euclidean windows

Stefania Cavallar Stefania.Cavallarecwi.nl

CWI, Kruislaan 413

P.O. Box 94079

1090 GB Amsterdam

The Netherlands

Franz Lemmermeyer franzlecsusm.edu

Univ. San Marcos

Department of Mathematics

333 South Twin Oaks Valley Road

San Marcos, CA 92096-0001 